\title{
Heterozygote Autosomal Dominant Mutation of STXBP1-Gene Inducing Encephalopathy with Global Developmental Delay, Atactic Movement Disorder and Focal Seizures in A 2-Years Old Boy
}

\author{
Stefan Bittmann*, Anne Weissenstein, Elisabeth Luchter, Elena Moschüring Alieva, Anne Weissenstein and Gloria \\ Villalon \\ Department of Pediatrics, Ped Mind Institute, Germany \\ *Corresponding author: Stefan Bittmann, Department of Pediatrics, Ped Mind Institute, Germany. \\ To Cite This Article: Stefan Bittmann, Heterozygote Autosomal Dominant Mutation of STXBP1-Gene Inducing Encephalopathy with Global \\ Developmental Delay, Atactic Movement Disorder and Focal Seizures in A 2-Years Old Boy. Am J Biomed Sci \& Res. 2019 - 6(5). AJBSR.MS.ID.001075. \\ DOI: 10.34297 /AJBSR.2019.06.001075.
}

Received: 继 December 14, 2019 ; Published: 眥 December 18, 2019

\section{Short Comment}

Heterozygote autosomal dominant mutation of STXBP1-gene induces global developmental delay and atactic movement disorder with focal seizures in a 2-years old boy. To date, only around 200 cases are known in world medical literature. The mutation and the outcome of children with this unusual mutation of STXBP1-gene in a 2 years old young boy in our department was the occasion to make a short comment.

STXPBP1 gene mutation can induce encephalopathy in children characterized by recurrences (epilepsy), abnormal brain function and intellectual delay. At least 200 individuals with this condition have been described in the medical literature. The first signs and symptoms are present in early childhood with the presence of intermittent seizures in form of infantile spasm. These spasms occur before the first year of life and consist of uncontrolled muscle contractions. Cases of myoclonic seizures, weak muscle tones in form of atonic seizures, absence seizures and forms of tonic-clinic seizures are well known. Patients with STXBP1 encephalopathy with epileptically seizures show different types of seizures. 25per cent of patient's refractory seizures and these seizures do not respond to anticonvulsive medications. STXBP1 encephalopathy associated with epilepsy includes disability of intellectual ability and often found in moderate forms. Patients and children with this gene mutation have delayed development of speech and walking. In a few patients, these skills never fully develop. Many newborns have feeding disabilities. Areas of brain tissue loss with atrophy of brain regions have been found on medical imaging. This condition is found in an autosomal dominant genetic pathway, which impresses one copy of the altered gene in each cell is enough to cause the disorder. Many of these mutations result from de novo mutations in the gene that occur during the formation of reproductive cells in an affected individuals' parent or in early embryonic development. The prevalence of STXBP1 encephalopathy with epilepsy is unknown. 